\title{
ECORREGION EJE CAFETERO: Una lectura desde los propósitos compartidos. ${ }^{1}$
}

\author{
COFFEE ECOREGION:
}

A Reading Based on Shared Purposes

Oscar Arango Gaviria ${ }^{2}$

COLOMBIA

Palabras Clave: Eco-región, territorio, Desarrollo regional, agendas.

Key words: Ecoregion, territory, regional development, agendas.

\section{Resumen}

Se describe el territorio de la Ecorregión y se identifican algunos de sus problemas; se resalta la dinámica de construcción de la Ecorregión coexistiendo con estructuras departamentales (completas e incompletas) y se subrayan algunas limitaciones del proceso. Luego, el artículo se ocupa de describir la progresiva búsqueda de acuerdos para el desarrollo sostenible en este territorio, llamando la atención de la forma como han aparecido desde 2001 diferentes acuerdos que se han expresado en Agendas y Declaraciones. Se culmina resaltando los aportes que las comunidades académicas regionales han realizado a este ejercicio de concertación.

\begin{abstract}
This paper describes the ecoregion territory and identifies some of its problems. The construction dynamics of the ecoregion is shown as an important issue, which is related to structures of different states (complete and incomplete). This paper explores some limitation of the process and describes the progressive search for agreements of sustainable development in this territory. Particularly, the different agreements, agendas and declarations that emerged since 2001 call our attention. Finally, the paper highlights the contributions of the regional academic communities to this common effort.
\end{abstract}

1 Ponencia en el VIII Seminario de la RIPPET. Manizales, 6 - 8 de agosto, 2009

2 Profesor Universidad Tecnológica de Pereira. Coordinador Proyectos Regionales, Red Alma Mater. Colombia 


\section{EL TERRITORIO}

La Ecorregión Eje Cafetero es el corazón geográfico de Colombia. De esta Ecorregión hacen parte, de manera completa, los departamentos de Caldas, Quindío y Risaralda. Pero también la integran el norte del Valle y el noroccidente del Tolima.

La región está situada en el centro del triángulo Bogotá - Cali - Medellín donde habita el $58 \%$ de la población total del país, se produce el $76 \%$ del producto interno bruto, se realiza el $75 \%$ del comercio y se ofrece el $73 \%$ de los servicios generales. Al Eje Cafetero lo cruzan ejes de desarrollo geo-económico como el corredor vial Buenaventura - Bogotá - Caracas, integrador del comercio entre el Atlántico y el Pacífico.

Desde el punto de vista ambiental, en este territorio se cuenta con parte de la cordillera Central, en sus dos laderas y con parte de la cordillera occidental, en su ladera oriental. También se suman las cuencas medias de los ríos Magdalena y Cauca. Se dispone de una extraordinaria biodiversidad, de una densa red hídrica y de un amplio sistema de áreas protegidas, todo lo cual conforma una importante plataforma natural para el desarrollo.

A lo largo de los ejes estructurantes naturales se levanta una compleja red de ciudades, que hoy nos permite hablar de la Ciudad Región Eje Cafetero. En nuestras zonas urbanas hoy habita el $76 \%$ de la población, lo cual supone nuevos desafíos para conseguir una adecuada articulación entre los procesos urbanos y rurales.

A pesar de sus favorables condiciones naturales y de ubicación, nuestra Ecorregión está hoy sumergida en una crisis social con las más altas tasas de desempleo del país y con un progresivo empobrecimiento, a lo cual se suma la llegada de desplazados por la violencia, y altos índices de inseguridad social. El actual colapso económico se ha encargado de colocar de presente la magnitud de nuestros retos para buscar, encontrar y aplicar nuevas alternativas de desarrollo.

\section{ECORREGIÓN EN MEDIO DE ESTRUCTURAS DEPARTAMENTALES}

El trabajo de construcción cultural, social y política de la Ecorregión Eje Cafetero encuentra una parte importante de su justificación en los problemas de ordenamiento territorial no resueltos de Colombia. Problemas que hacen referencia, tanto a una estructura político administrativa que no propicia la descentralización y la autonomía de las entidades territoriales, como a una relación sociedad-naturaleza que termina privilegiando el uso no sostenible de los recursos naturales y hace a un lado las opciones del desarrollo endógeno.

Desde el siglo XIX en este territorio los límites político administrativos han tenido diversos acomodamientos. En 1886 hacíamos parte del Cauca y de Antioquia. Al despuntar el siglo XX el presidente Reyes dio paso al

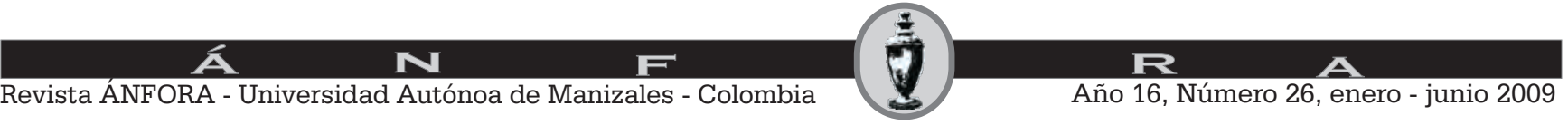


Mapa Nº 1. Ecorregión Eje Cafetero.

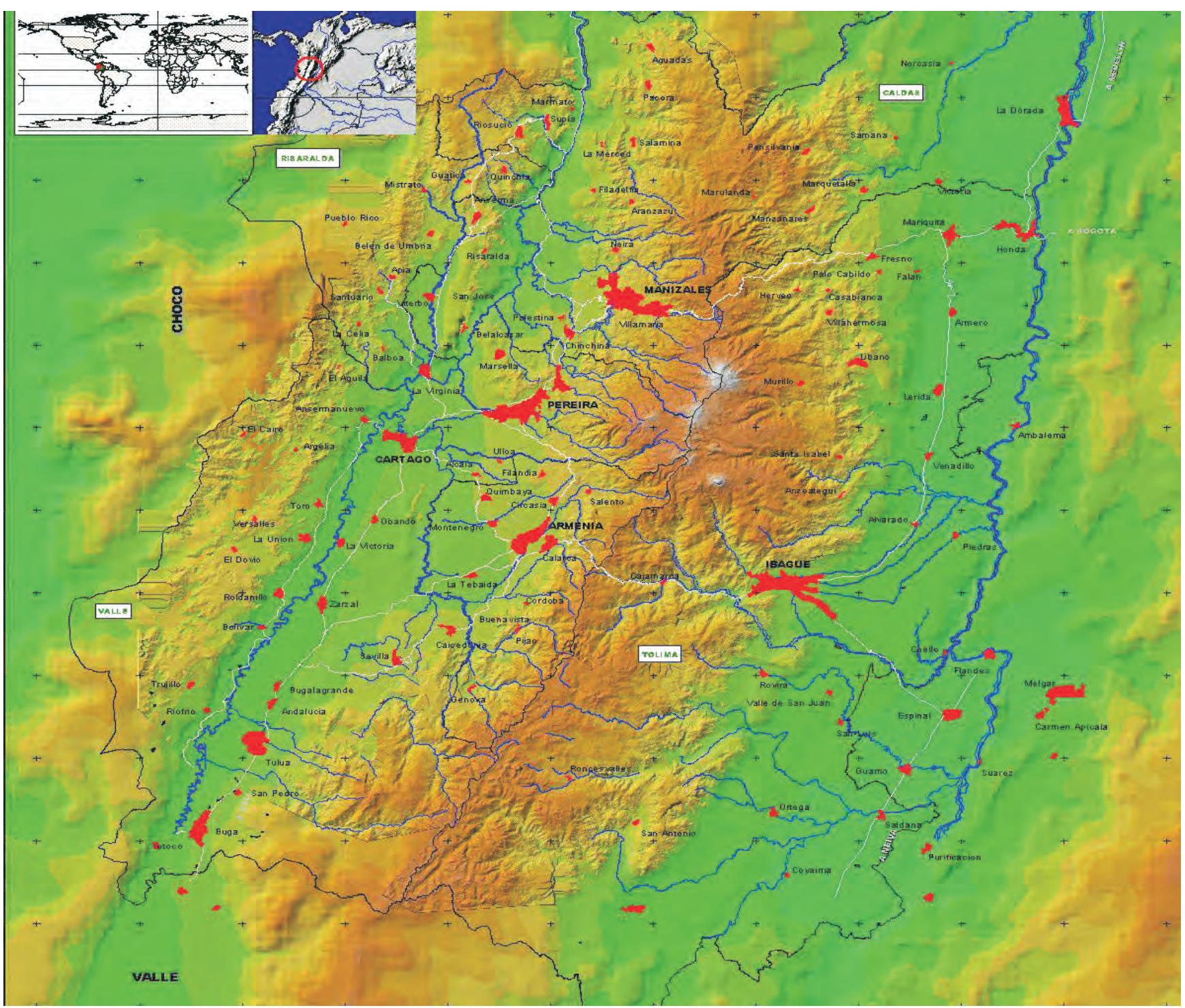

Fuente: Sistema de Información Regional. SIR. Pereira. 2004

departamento de Caldas que, al finalizar los años 60 sintió el impacto de la departamentalitis que invadió al país y de sus entrañas surgieron Quindío y Risaralda.

Más recientemente hemos presenciado movimientos en diferentes direcciones. Desde declaraciones de interés regional por parte de gobernadores, alcaldes, concejales y diputados, ${ }^{3}$ hasta movimientos locales o subregionales de orden
3 Por ejemplo, las relacionadas con un acuerdo de gobernadores para trabajar conjuntamente la problemática de la infancia, la adolescencia y la juventud, o el proceso que adelantan los concejales para atender conjuntamente 
político que expresan su inconformidad por el abandono en que los tienen sus respectivas gobernaciones y amenazan con una eventual separación para pasarse a otro departamento.

Esta última dinámica que ya completa un par de décadas involucra municipios de Caldas que quisieran ser parte de Risaralda; localidades del Tolima que tocan las puertas de Caldas o municipios del norte del Valle que llaman la atención para sumarse al Quindío. Ejemplos de estos se pueden citar a lo largo y ancho de Colombia, y no hacen más que ratificar la fragilidad y falta de real legitimidad de nuestro ordenamiento territorial en su dimensión político administrativa. Pero también son una prueba de iniciativas que no reconocen las causas estructurales del problema y confían en una eventual solución reducida a cambiar de departamento, sin que exista ninguna garantía de mayores recursos o de una gestión local más descentralizada y autónoma.

Aún más, debe reconocerse que prácticamente en todos los casos, pero con mayor razón en los ejemplos de los departamentos creados durante los años 60, se trató de la configuración de límites político administrativos acordados en las mesas de negociación de dirigentes políticos y gremiales que trazaron líneas fronterizas y sumaron o restaron municipios dependiendo de su particular interés político partidista. ${ }^{4}$ No de otra manera podrían explicarse las intrincadas líneas divisorias de nuestros mapas departamentales.

En la reconfiguración departamental no aparecieron, en absoluto, consideraciones alrededor de ecosistemas estratégicos, manejo de la biodiversidad, recurso hídrico o de la plataforma natural para promover el desarrollo.

Por lo demás, conviene recordar que la Asamblea Constituyente de 1991, a pesar de sus avances en materia de descentralización y autonomía territorial, dejó sin resolver los asuntos estructurales para propiciar un reordenamiento territorial del país y, de manera particular, aplazó la búsqueda de acuerdos para una reorganización de los denominados niveles intermedios entre el Estado central y el municipio. Ese nivel intermedio continuó siendo el departamento heredado desde la Constitución de 1886.

Aunque no es el tema central de estas reflexiones, sea este el momento de reconocer que es inocultable la urgencia de un acuerdo político nacional para dotar al país de una Ley Orgánica de Ordenamiento Territorial, es decir, de una política de Estado que atienda, desde los procedimientos democráticos, la construcción de una nueva arquitectura tanto de la dimensión territorial institucional del Estado, como de las relaciones entre la sociedad y la naturaleza que hagan sostenible el desarrollo para las presentes y futuras generaciones de colombianos.

Pero volviendo a nuestro tema, debe recordarse que hemos venido trabajando bajo unas circunstancias político - administrativas que, a lo sumo, favorecen la asociación de dos o más departamentos, pero que no contemplan la posibilidad
4 Cfr. RODRIGUEZ, Jahir y otros. Estado, política y gremios en la construcción de Risaralda. UTP, Pereira, 1987

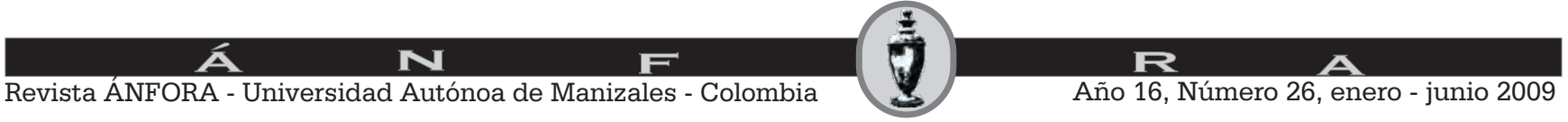


de arreglos institucionales entre fragmentos de departamentos o entre estos y estructuras departamentales completas.

\section{EN LA BÚSOUEDA DE UN NORTE PARA LA ECORREGIÓN.}

Nuestro más inmediato antecedente en la construcción de esta Ecorregión, se remonta 10 años atrás, cuando en 1999 el trabajo y el apoyo de miles de colombianos permitieron adelantar las tareas propias de la reconstrucción del Eje Cafetero.

Con el apoyo, entre otros actores, de diferentes universidades fue posible atender, no solo tareas específicas de la emergencia derivada del terremoto, sino también las relacionadas con la planificación de este territorio. En su momento se construyó, en medio del drama y la desolación, la Agenda para la reconstrucción social, ${ }^{5}$ un instrumento destinado a garantizar que efectivamente los temas sociales estuvieran siempre en primer lugar de atención.

Surgieron entonces muchos de los planes zonales y sectoriales, y lo más importante, se construyó una lectura integral del proceso que dio origen a los lineamientos de ordenamiento territorial con énfasis en amenazas, ya no sólo para los 28 municipios objeto del desastre, sino para los 92 municipios que desde entonces conforman la Ecorregión Eje Cafetero.

Contando con ese insumo, vinieron luego las reflexiones que fundamentaron en 2001 la primera versión de la Agenda para el Desarrollo Sostenible de la Ecorregión. Este documento, fruto de un amplio proceso de deliberación pública, identifica tendencias positivas y negativas, se ocupa de las potencialidades, diseña escenarios futuros y contiene una estructura visional que articula cuatro dimensiones básicas: 1. La gobernabilidad democrática; 2. $\mathrm{El}$ ambiente protegido; 3. La cultura, la identidad y la pertinencia, y 4. El desarrollo económico, la equidad social y la competitividad.

La Agenda citada precisa los objetivos básicos acordados, entre los cuales pueden destacarse:

- Trabajar por la gobernabilidad democrática, la convivencia y la paz;

- Asumir el Desarrollo Sostenible, como dinamizador de la productividad y manejo de los recursos naturales para generaciones futuras;

- Reconocer el fortalecimiento del tejido socio-cultural, como forma de expresión, comunicación y solidaridad;

- Promover la diversificación productiva de mercados, como medio para el mejoramiento de la calidad de vida de los habitantes;

- Consolidar el desarrollo de la infraestructura regional;

5 FOREC-Fundación Espiral. Agenda para la reconstrucción social del Eje Cafetero. Armenia, 1999

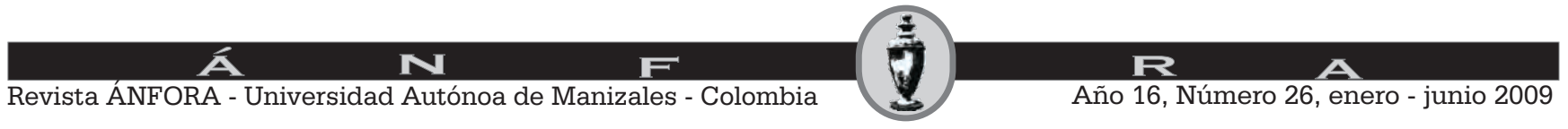


- Mejorar la valoración y fomento de bienes y servicios ambientales y la conservación de la biodiversidad, y

- Continuar la tarea del fortalecimiento del conocimiento mediante la investigación y la educación.

Entre 2001 y 2005 se retroalimentó esta Agenda; se avanzó en la concertación de prioridades regionales y se definieron líneas de acción visionales e instrumentales, cada una de las cuales se ha estado acompañando de sus respectivos proyectos.

Entre las líneas visionales se pueden citar: Reconversión socio ambiental de sistemas productivos rurales; Producción más limpia; Gestión del hábitat y desarrollo humano sostenible; Manejo integral de agua; Protección, conservación y restauración de la biodiversidad; Planificación y ordenamiento del territorio, y Conectividad Regional, donde aparece con toda claridad el rol que podemos y debemos jugar quienes trabajamos por la construcción de comunidades académicas puestas al servicio de la Ecorregión.

En los últimos cinco años el Gobierno nacional ha aprobado diferentes documentos CONPES que apuntan a dar respuesta a problemas específicos, pero se carece de una lectura integral del futuro de la Ecorregión y por ello se ha venido trabajando en la formulación de una propuesta que, al ser aprobada por el Consejo Nacional de Política Económica y Social redefina las relaciones Región-Nación, precise los programas estratégicos que serán respaldados por los diferentes Ministerios e institutos descentralizados, y garantice un acuerdo de cofinanciación del desarrollo para estos 92 municipios.

Es decir, hemos acordado gestionar un Documento CONPES ${ }^{6}$ que maneje una perspectiva de desarrollo sostenible para la Ecorregión y ahora trabajamos en la construcción de condiciones político institucionales que hagan viable esa iniciativa.

Mientras nos movemos en la dirección citada, otros acuerdos han sido suscritos por diversos actores. Por ejemplo, en 2006 los gobernadores de los cinco departamentos hicieron pública su intención de realizar gestiones conjuntas en procura del desarrollo de este territorio y convinieron en la creación de una mesa técnica integrada por los Secretarios de Planeación. ${ }^{7}$

En 2007, en un ejercicio sin antecedentes en la región, se realizó en Armenia, Cartago, Manizales y Pereira el foro itinerante que se denominó UniversidadEmpresa-Estado construyen región desde el Eje Cafetero. Este evento recordó que desde la Academia, la Empresa privada, el Estado y la sociedad civil, estamos en capacidad de generar conocimiento que aporte al desarrollo regional sostenible y a la convivencia pacífica. Los participantes exhortaron, entre otros propósitos, a generar espacios de trabajo común que impulsen el desarrollo local sostenible y la convivencia pacífica para convertir nuestra región en un actor competitivo a nivel global. ${ }^{8}$
6 Cfr. CASTAÑO, Diego. Propuesta de Documento CONPES para la Ecorregión Eje Cafetero. Tesis de Grado. UTP. Pereira, 2007

7 Cfr. Conclusiones de la Cumbre de Gobernadores. Pereira, enero 26, 2006

8 Cfr. Alma Mater y otros. Universidad +Empresa +Es tado construyen región en el Eje Cafetero. Ver Declaración final. Pereira, 2008

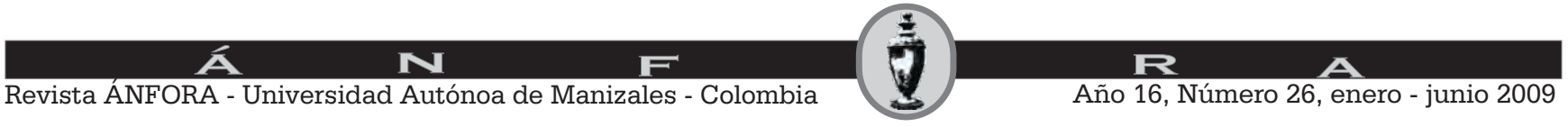


En 2008, en el marco de una evaluación de los 15 años del Sistema Nacional Ambiental, quienes hicieron parte de una jornada de trabajo sobre el desarrollo sostenible de la Ecorregión, suscribieron una declaración que incorporó lineamientos de trabajo alrededor de la gestión integral del recurso hídrico; el Sistema regional de Áreas Protegidas; el Paisaje Cultural Cafetero; el café y el medio ambiente, lo mismo que sobre la institucionalidad que debe servir de soporte a la perspectiva integral y de largo plazo del desarrollo. ${ }^{9}$

Más recientemente, en abril de 2009, y admitiendo que el agua debe representar un inequívoco eje articulador en la construcción de la Ecorregión, luego de un intenso ejercicio de reflexión académica y política, se expidió una Declaración que llama a fortalecer el trabajo de ordenación y manejo de las cuencas, participando de la discusión nacional sobre la política pública que debe orientar la ordenación y manejo de cuencas; atendiendo con particular interés el trabajo de las cuencas compartidas por dos o más Corporaciones Autónomas; propiciando escenarios democráticos para el trabajo de la revisión de los planes y esquemas de ordenamiento territorial; buscando alianzas con organizaciones ambientalistas de ámbito nacional e internacional para la consolidación de acciones de conservación y ordenamiento ambiental territorial.

Esta Declaración de Ibagué llama a crear o fortalecer una red interinstitucional como espacio permanente de discusión y análisis de temas relacionados con la gestión integral en cuencas hidrográficas; e invita a afianzar los procesos y estrategias de investigación-gestión que sobre ordenación y manejo ambiental de cuencas hidrográficas se adelanten y/o requieran en la Ecorregión, facilitando la movilidad académica y el diálogo de saberes entre grupos de investigación pertinentes, autoridades ambientales, y por supuesto sabios lugareños que participan de instancias y planes de ordenación de cuencas. ${ }^{10}$

En fin, en este proceso nos hemos dotado de una visión que a la letra dice: "en el año 2019 la Ecorregión Eje Cafetero será un territorio integrado política, social y económicamente, arraigado en sus culturas, socialmente equitativo, diversificado en su producción, ambientalmente sostenible y competitivo, donde se garantiza la paz y la seguridad ciudadana, planificado con base en sus potencialidades territoriales y valores ambientales, hábil en la concertación de prioridades regionales y efectivo en su realización, ambientalmente protegido, biodiverso y con una sólida infraestructura para la conectividad regional".

\section{APORTES DESDE LA ACADEMIA}

Antes de terminar debe reconocerse que de este ejercicio de construcción de la Ecorregión han participado muchos actores estatales, de la sociedad civil, del sector privado y de la academia.

Diferentes grupos de investigación de las universidades de la Ecorregión, cada uno a su manera, han contribuido a documentar, a entender y a plantear alternativas de solución para enfrentar los graves problemas estructurales y coyunturales que hoy nos aquejan.
9 Cfr. Alma Mater y otros. Hacia el desarrollo sostenible en la Ecorregión Eje Cafetero. Ver: Declaración de La Casona. Pereira, 2009

10 Cfr. Alma Mater y otros. Declaración de Ibagué. 2009

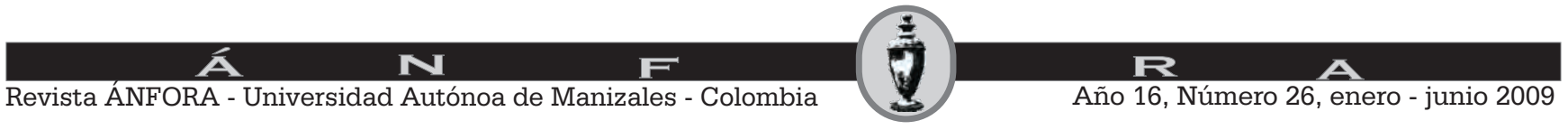


En estas materias siempre faltará camino por recorrer, pero ahora tenemos mejores criterios e información para comprender los temas relacionados con la gobernabilidad democrática o con las migraciones internacionales; con el desarrollo tecnológico o con el trabajo en redes; con las razones de nuestro conflicto armado o con las posibilidades de nuestros bienes y servicios ambientales.

Por supuesto, parte importante del esfuerzo derivado de este Seminario debería orientarse a gestionar imaginativas e innovadoras formas de concertación interinstitucional e internacional que permitan consolidar las capacidades de nuestras comunidades académicas regionales.

El fortalecimiento de la Red Iberoamericana que hoy nos agrupa pasa, precisamente, por continuar tejiendo sin prisa, pero sin pausa, el entramado de las más diversas formas de convenios y acuerdos interinstitucionales por cuyo intermedio la Ecorregión le aporte al desarrollo de la Nación colombiana, y esta se sume al escenario de las soluciones internacionales.

\section{Bibliografía.}

ALMA MATER y otros. Universidad+Empresa+Estado construyen región en el Eje Cafetero. Ver Declaración final. Pereira, 2008

ALMA MATER y otros. Hacia el desarrollo sostenible en la Ecorregión Eje Cafetero. Ver: Declaración de La Casona. Pereira, 2009

ALMA MATER y otros. Declaración de Ibagué. 2009

CASTAÑO, Diego. Propuesta de Documento CONPES para la Ecorregión Eje Cafetero. Tesis de Grado. UTP. Pereira, 2007

Conclusiones de la Cumbre de Gobernadores. Pereira, enero 26, 2006

FOREC-Fundación Espiral. Agenda para la reconstrucción social del Eje Cafetero. Armenia, 1999

RODRÍGUEZ, Jahir y otros. Estado, política y gremios en la construcción de Risaralda. UTP, Pereira, 1987

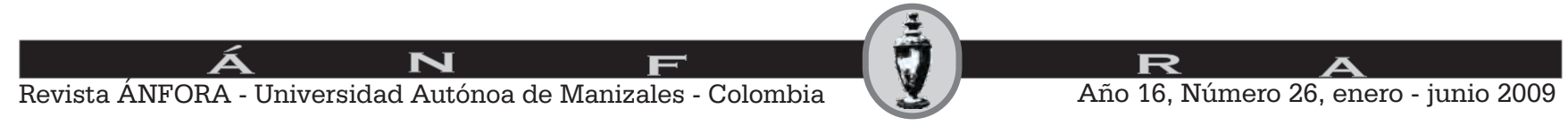

